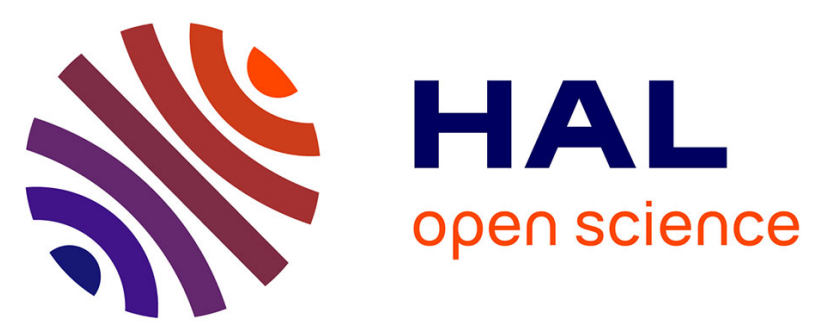

\title{
Symmetry analysis and exact model for the elastic, piezoelectric, and electronic properties of inhomogeneous and strained wurtzite quantum nanostructures \\ Jacky Even
}

\section{- To cite this version: \\ Jacky Even. Symmetry analysis and exact model for the elastic, piezoelectric, and electronic properties of inhomogeneous and strained wurtzite quantum nanostructures. Applied Physics Letters, 2009, 94, pp.102105. 10.1063/1.3097232 . hal-00492273}

\section{HAL Id: hal-00492273 \\ https://hal.science/hal-00492273}

Submitted on 15 Jun 2010

HAL is a multi-disciplinary open access archive for the deposit and dissemination of scientific research documents, whether they are published or not. The documents may come from teaching and research institutions in France or abroad, or from public or private research centers.
L'archive ouverte pluridisciplinaire HAL, est destinée au dépôt et à la diffusion de documents scientifiques de niveau recherche, publiés ou non, émanant des établissements d'enseignement et de recherche français ou étrangers, des laboratoires publics ou privés. 


\title{
Symmetry analysis and exact model for the elastic, \\ piezoelectric, electronic properties of inhomogeneous and strained wurtzite quantum nanostructures
}

\author{
J. Even ${ }^{\text {a) }}$ \\ FOTON-INSA Laboratory, UMR 6082 au CNRS, INSA de Rennes, 20 Avenue des Buttes de \\ Coësmes, CS 14315, 35043 Rennes Cedex, France
}

\begin{abstract}
.
A symmetry analysis and a semi analytical exact model are proposed to describe the mechanical, piezoelectric and electronic properties of strained wurtzite quantum nanostructures (QNs) with axial symmetry. An expression of the piezoelectric polarization is given as a function of inhomogeneous strains. The 3D 8x8 strained k.p Hamiltonian is reduced to $2 \mathrm{D}$ using the total angular momentum representation. When the spin-orbit coupling is neglected, the Hamiltonians are reduced to $1 \times 1$ and $3 \times 3$ Hamiltonians for the states in the S-shell. For all the other shells, the four-fold degeneracy is demonstrated. Simulations are performed for InN/GaN quantum dots (QDs).
\end{abstract}

P.A.C.S. 71.20.Nr, 73.21.La, 77.65.Ly

\footnotetext{
${ }^{\text {a)} E l e c t r o n i c ~ m a i l ~: ~ j a c k y . e v e n @ i n s a-r e n n e s . f r ~}$
} 
A general model for bulk strained wurtzite (WZ) semiconductors ${ }^{1,2}$ has been extended later to quantum wells $(\mathrm{QWs})^{3}$ and quantum dots $(\mathrm{QDs})^{4,5}$. A block-diagonalization of the valence band (VB) Hamiltonian is possible for a strained bulk sample ${ }^{1,2}$ or a homogeneous and unstrained cylindrical quantum nanostructure $\mathrm{QN}^{6}$. The simulation of electronic and optical properties requires a precise description of elastic and piezoelectric effects ${ }^{4,5}$. Moreover, the continuous variation of chemical composition around the nanostructure has often to be included into the theoretical description ${ }^{5}$. The aim of this paper is to propose a complete symmetry analysis and a semi analytical model for mechanical, piezoelectric and electronic properties of strained and inhomogeneous WZ QNs with a cylindrical geometry by analogy to QNs in zinc-blende lattice ${ }^{7,8}$. Symmetry properties are used as much as possible to simplify the problem.

QN geometries with axial symmetry are studied in this work. The $\mathrm{C}_{\infty \mathrm{v}}$ axis for the geometry is perpendicular to the (0001) plane (parallel to the [0001] c axis). Physical parameters for InN and $\mathrm{GaN}$ materials ${ }^{9,10}$ are used for the WZ lattice ${ }^{3,4}$. If the strain field is calculated using a continuum mechanical model (elasticity), the geometry of the deformed inhomogeneous QN still corresponds to the $\mathrm{C}_{\infty \mathrm{v}}$ symmetry. This effect is associated to the $C_{66}=\left(C_{11}-C_{12}\right) / 2$ relation between elastic constants. Five independent parameters $\left(\boldsymbol{C}_{11}, \boldsymbol{C}_{12}, C_{13}, C_{33}, \boldsymbol{C}_{44}\right)$ describe the mechanical properties of the WZ lattice which is related to the "transverse isotropic" materials class. The components of the strain tensor using the cylindrical representation $(\boldsymbol{r}, \boldsymbol{\varphi}, \boldsymbol{z})$ depend only on four quantities $\mathcal{E}_{r r}(r, z), \varepsilon_{\varphi \varphi}(r, z), \varepsilon_{z z}(r, z), \mathcal{\varepsilon}_{r z}(r, z)$ in this case $^{7,8}$. 2D calculations can then be performed in cylindrical coordinates $(\boldsymbol{r}, \boldsymbol{z})$ to evaluate the strains but also the piezoelectric potential. The polarization $\vec{P}_{W Z}$ (including the pyroelectric $\vec{P}_{S P}$, and piezoelectric radial $\vec{P}_{r a d}$ and vertical $\vec{P}_{v e r t}$ contributions) is:

$$
\vec{P}_{W Z}=2 e_{15} \varepsilon_{r z} \vec{u}_{r}+\left(P_{S P}+e_{31}\left(\varepsilon_{r r}+\varepsilon_{\varphi \varphi}\right)+e_{33} \varepsilon_{z z}\right) \vec{u}_{z}
$$


We may notice first, that this expression is valid for inhomogeneous materials and QNs. It corresponds to an axially symmetric electric potential $V(r, z)$ (axial 2D Poisson equation). By comparison, a symmetry breaking always occurs in the $\mathrm{ZB}$ case for shear strains (reduction to $\left.\mathrm{C}_{2 \mathrm{v}}\right)$ whatever the symmetry initially considered $\left(\mathrm{C}_{\infty \mathrm{v}}\right.$ or $\left.\mathrm{C}_{4 \mathrm{v}}\right)$ for the strain field ${ }^{7}$. Results for a truncated conic InN/GaN QD (full height, radius and truncation height are respectively equal to 8,4 and $2 \mathrm{~nm}$ ) are shown on figure 1 . The total electric potential (figure 1-a) is due to the three contributions: $\vec{P}_{\text {rad }}$ (figure 1-b), $\vec{P}_{\text {vert }}$ (figure 1-c) and $\vec{P}_{S P}$. The $\vec{P}_{S P}$ contribution is very small ${ }^{5}$ and not shown on figure 1 . The electric potential depends mainly on $\vec{P}_{v e r t}$ but the contribution due to $\vec{P}_{\text {rad }}$ may be opposite. The influence of $\vec{P}_{\text {rad }}$ is less important in QD when the aspect ratio (height/radius) is small. Figure 2 presents the variation of the conduction band (CB) ground state $(\mathrm{GS}) \quad\left(\mathrm{F}_{\mathrm{za}}=1 / 2\right.$, see the description of the electronic states) energy as a function of the truncation height, when the electric potential is: a) calculated with $\vec{P}_{r a d}$, b) calculated with the total polarization $\vec{P}_{W Z}$, c) equal to zero, d) calculated with $\vec{P}_{S P}$ and e) calculated with $\vec{P}_{v e r t}$. The effect of $\vec{P}_{S P}$ is very small. For this particular geometry, the Stark energy shifts associated to $\vec{P}_{r a d}$ and $\vec{P}_{v e r t}$ are opposite. The wave function and energy of this state depend on the shape of QD.

For the study of the electronic properties, we start from the $8 x 8$ k.p Hamiltonian ${ }^{1}$ used previously for inhomogeneous and strained QNs in Cartesian coordinates ${ }^{5}$ : $H=\left(\begin{array}{cccccccc}A & -i V^{*} & i V & i U & 0 & 0 & 0 & 0 \\ i V & F_{+} & -K^{*} & -H^{*} & 0 & 0 & 0 & 0 \\ -i V^{*} & -K & F_{-} & H & 0 & 0 & 0 & \sqrt{2} \Delta_{2} \\ -i U & -H & H^{*} & \lambda & 0 & 0 & \sqrt{2} \Delta_{2} & 0 \\ 0 & 0 & 0 & 0 & A & i V & -i V^{*} & i U \\ 0 & 0 & 0 & 0 & -i V^{*} & F_{+} & -K & H \\ 0 & 0 & 0 & \sqrt{2} \Delta_{2} & i V & -K^{*} & F_{-} & -H^{*} \\ 0 & 0 & \sqrt{2} \Delta_{2} & 0 & -i U & H^{*} & -H & \lambda\end{array}\right)$ 
All the terms of the Hamiltonian can be in fact given exactly in the cylindrical representation including chemical inhomogeneities, strains and the electric potential:

$$
\begin{aligned}
& A=E_{c}-e V(r, z)+\frac{\hbar^{2}}{2 m_{0}} A_{2}^{\prime} k_{+} k_{-}+\frac{\hbar^{2}}{2 m_{0}} A_{1}^{\prime} k_{z}^{2}+a_{2}\left[\varepsilon_{r r}+\varepsilon_{\phi \phi}\right]+a_{l} \varepsilon_{z z}, V=\frac{P_{2} k_{-}}{\sqrt{2}}, U=P_{1} k_{z} \\
& F \pm=\Delta_{l} \pm \Delta_{2}+\lambda+\theta, \theta=\frac{\hbar^{2}}{2 m_{0}}\left[A_{4}+\frac{P_{1}^{2}}{2 E_{g}}\right] k_{+} k_{-}+\frac{\hbar^{2}}{2 m_{0}}\left[A_{3}-\frac{P_{2}^{2}}{E_{g}}\right] k_{z}^{2}+D_{4}\left[\varepsilon_{r r}+\varepsilon_{\phi \phi}\right]+D_{3} \varepsilon_{z z} \\
& \lambda=E_{v}-e V(r, z)+\frac{\hbar^{2}}{2 m_{0}} A_{2} k_{+} k_{-}+\frac{\hbar^{2}}{2 m_{0}}\left[A_{1}+\frac{P_{2}^{2}}{E_{g}}\right] k_{z}^{2}+D_{2}\left[\varepsilon_{r r}+\varepsilon_{\phi \phi}\right]+D_{l} \varepsilon_{z z} \\
& K=\frac{\hbar^{2}}{2 m_{0}}\left[A_{5}+\frac{P_{1}^{2}}{2 E_{g}}\right] k_{+}^{2}+D_{5}\left[\varepsilon_{r r}-\varepsilon_{\phi \phi}\right] e^{2 i \phi}, A_{1}^{\prime}=\frac{1}{m_{z}}-\frac{P_{1}^{2}}{E_{g}}, A_{2}^{\prime}=\frac{1}{m_{x y}}-\frac{P_{2}^{2}}{E_{g}}, \Delta_{2}=\frac{\Delta_{S O}}{3} \\
& H=\frac{\hbar^{2}}{2 m_{0}}\left[A_{6}+\frac{P_{1} P_{2}}{\sqrt{2} E_{g}}\right] k_{+} k_{z}-\frac{i \hbar^{2}}{2 m_{0}} A_{7} k_{+}+D_{6} \varepsilon_{r z} e^{i \phi} \text { (in most cases } A_{7}=0^{5} \text { ) }
\end{aligned}
$$

where $\Delta_{S O}$ is the spin-orbit coupling parameter and $k_{ \pm}=-i e^{ \pm i \varphi}\left(\frac{\partial}{\partial r} \pm \frac{i}{r} \frac{\partial}{\partial \varphi}\right)$

The 8 x8 QNs Hamiltonian is then reduced to $2 \mathrm{D}$ in a $\boldsymbol{F}_{z}$ representation $\left(\boldsymbol{F}_{z}=\boldsymbol{J}_{z}+\boldsymbol{L}_{\boldsymbol{Z}}\right.$ is the total angular momentum), following the Sercel-Vahala method ${ }^{11,12}$. The basis is constructed in a product form $\left|\boldsymbol{J}_{,} \boldsymbol{J}_{z}\right\rangle\left|\boldsymbol{L}_{z}=\boldsymbol{F}_{z}-\boldsymbol{J}_{z}\right\rangle$ where the first factor corresponds to one of the 8 band-edge Bloch functions related to the bands of the bulk material and the second factor $\left|L_{z}=F_{z}-J_{z}\right\rangle=\frac{1}{\sqrt{2 \pi}} e^{i L_{z} \varphi} f(r, z)$ to the envelope functions adapted to the usual 1-band cylindrical representation $\left(\mathrm{C}_{\infty \mathrm{v}}\right.$ symmetry). Spin degeneracy is associated to $\pm F_{z}$.

In ref [4], it was pointed out an additional simplification is obtained when neglecting the spin-orbit coupling $\Delta_{S O}=0$. It is a reasonable approximation for most nitride compounds in a wurtzite lattice. In that case $F_{+}=F_{-}=F$ and the $8 \times 8$ Hamiltonian is decoupled into two $4 \times 4$ Hamiltonians. The $4 \times 4$ problems can be solved yielding separated eigenvectors. The degeneracy associated to $\pm F_{z}$ still exists. An additional degeneracy is introduced by the 
$\Delta_{S O}=0$ approximation. For the upper (lower) $4 \times 4$ block, the same eigenvalue is obtained for $F_{z a}$ and $F_{z b}$ providing that $F_{z a}+F_{z b}=1\left(F_{z a}+F_{z b}=-1\right)$. If $F_{z a} \neq 1 / 2$, the corresponding fourfold degenerate eigenvectors are given by:

$$
\begin{gathered}
\left.\left|\psi_{F_{z a}}^{U}\right\rangle=\frac{1}{\sqrt{2 \pi}}\left|\begin{array}{l}
e^{i\left(F_{z a}-1 / 2\right) \varphi} f_{1}(r, z) \\
e^{i\left(F_{z a}-3 / 2\right) \varphi} f_{2}(r, z) \\
e^{i\left(F_{z a}+1 / 2\right) \varphi} f_{3}(r, z) \\
e^{i\left(F_{z a}-1 / 2\right) \varphi} f_{4}(r, z)
\end{array}\right| \psi_{1-F_{z a}}^{U}\right\rangle=\frac{1}{\sqrt{2 \pi}} \mid \begin{array}{l}
e^{i\left(-F_{z a}+1 / 2\right) \varphi} f_{I}(r, z) \\
-e^{i\left(-F_{z a}-1 / 2\right) \varphi} f_{3}(r, z) \\
-e^{i\left(-F_{z a}+3 / 2\right) \varphi} f_{2}(r, z) \\
e^{i\left(-F_{z a}+1 / 2\right) \varphi} f_{4}(r, z)
\end{array} \\
\left.\left|\psi_{-F_{z a}}^{L}\right\rangle=\frac{1}{\sqrt{2 \pi}}\left|\begin{array}{l}
-e^{i\left(-F_{z a}+1 / 2\right) \varphi} f_{1}(r, z) \\
e^{i\left(-F_{z a}+3 / 2\right) \varphi} f_{2}(r, z) \\
e^{i\left(-F_{z a}-1 / 2\right) \varphi} f_{3}(r, z) \\
-e^{i\left(-F_{z a}+1 / 2\right) \varphi} f_{4}(r, z)
\end{array}\right| \psi_{F_{z a}-1}^{L}\right\rangle=\frac{1}{\sqrt{2 \pi}} \mid \begin{array}{l}
e^{i\left(F_{z a}-1 / 2\right) \varphi} f_{l}(r, z) \\
e^{i\left(F_{z a}+1 / 2\right) \varphi} f_{3}(r, z) \\
e^{i\left(F_{z a}-3 / 2\right) \varphi} f_{2}(r, z) \\
e^{i\left(F_{z a}-1 / 2\right) \varphi} f_{4}(r, z)
\end{array}
\end{gathered}
$$

In a recent $\mathrm{study}^{16}$, it was argued (by comparison to tight-binding results) that, the neglect of the spin-orbit coupling should remove the p-shell splitting. The $\mathrm{p}$-shell splitting predicted for QNs with hexagonal shapes in ref. 4 should then correspond to numerical artefacts. Our results for $F_{z a}=3 / 2$ are in agreement with the discussion presented in ref. 17 .

The particular case $F_{z a}=1 / 2$ corresponds to the S-shell. A two-fold degeneracy is obtained by setting first $\left|\psi_{F_{z a}}^{U}\right\rangle=-\left|\psi_{1-F_{z a}}^{U}\right\rangle:\left|\psi_{1 / 2}^{U}\right\rangle_{1}=\frac{1}{\sqrt{2 \pi}} \mid \begin{gathered}0 \\ e^{-i \varphi} f_{2}(r, z) \\ e^{i \varphi} f_{2}(r, z) \\ 0\end{gathered}$. In this first case, there is only one independent envelope function $f_{2}(r, z)$, which can be determined from a simple $1 \mathrm{x} 1$ block $H_{1 x 1}=F-K_{t}$. All the states determined from this equation are then in the S-shell of the VB. More, the wave functions do not contain any contributions from $|S\rangle$ and $|Z\rangle$ orbitals whereas the $\frac{1}{\sqrt{2}}|(X-i Y)\rangle$ and $\frac{1}{\sqrt{2}}|(X+i Y)\rangle$ orbitals have equal weights $(50 \%)$. In ref. 13 and 14, two-fold degenerate hole states are obtained. In that study, the $|S\rangle$ contribution is found equal to zero and the $|Z\rangle$ contribution is very small $(\sim 0.1 \%)$. This non-zero contribution may be either related to a numerical artefact, or to the fact that the geometry is 
only axially symmetric (atomistic effect) or to the theoretical method itself (tight binding instead of k.p) although s, px, py and pz atomic states are used. In ref. 4, a one-band effectivemass Hamiltonian is proposed for the holes to predict approximately the hole GS energy. Using the definitions of the parameters given previously, this approximate equation yields:

$$
\begin{aligned}
& H_{1 x l_{\text {appr }} .}=E_{v}+\Delta_{l}-e V(r, z)+\frac{\hbar^{2}}{2 m_{0}}\left(A_{2}+A_{4}-A_{5}\right) k_{t}^{2}+\frac{\hbar^{2}}{2 m_{0}}\left(A_{1}+A_{3}\right) k_{z}^{2} \\
& \ldots+\left(D_{2}+D_{4}-D_{5}\right)\left[\varepsilon_{r r}+\varepsilon_{\phi \phi}\right]+\left(D_{1}+D_{3}\right) \varepsilon_{z z}
\end{aligned}
$$

It is apparently almost equivalent to our exact 1x1 Hamiltonian: $H_{1 x l}=F-K_{t}=H_{1 x l_{a p p r} .}-2 D_{5} \varepsilon_{\phi \phi}$ where $K_{t}=\frac{\hbar^{2}}{2 m_{0}}\left[A_{5}+\frac{P_{1}^{2}}{2 E_{g}}\right] k_{t}^{2}+D_{5}\left[\varepsilon_{r r}-\varepsilon_{\phi \phi}\right]$.

This error introduced by missing the $-2 D_{5} \varepsilon_{\phi \phi}$ term is however important in our case. A twofold degeneracy is also obtained with $\left|\psi_{F_{z a}}^{U}\right\rangle=\left|\psi_{l-F_{z a}}^{U}\right\rangle:\left|\psi_{1 / 2}^{U}\right\rangle_{2}=\frac{1}{\sqrt{2 \pi}} \mid \begin{gathered}f_{1}(r, z) \\ e^{-i \varphi} f_{2}(r, z) \\ -e^{i \varphi} f_{2}(r, z) \\ f_{4}(r, z)\end{gathered}$

In this second case, the three envelope functions can be determined from reduced $3 \times 3$ blocks.

The wave function in the upper block $\left|\psi_{F_{z}}^{U}\right\rangle_{3 \times 3}=\frac{1}{\sqrt{2 \pi}} \mid \begin{gathered}f_{1}(r, z) \\ \sqrt{2} f_{2}(r, z) \\ f_{4}(r, z)\end{gathered}$ is indeed a function of the $\left(u_{1,0},|B\rangle=\frac{1}{\sqrt{2}}\left(e^{-i \varphi} u_{2,0}-e^{i \varphi} u_{3,0}\right), u_{4,0}\right)$ vectors : $H_{3 \times 3}^{U}=\left(\begin{array}{ccc}A & -i V_{t} & i U \\ i V_{t} & F+K_{t} & -\sqrt{2} H_{t} \\ i U & -\sqrt{2} H_{t} & \lambda\end{array}\right)$ where $H_{t}=\frac{\hbar^{2}}{2 m_{0}}\left[A_{6}+\frac{P_{1} P_{2}}{\sqrt{2} E_{g}}\right] k_{t} k_{z}+D_{6} \varepsilon_{r z}$

All the states in the S-shell of the CB correspond to this case. It is then not surprising that the CB GS contains significant contributions from the VB states of the bulk materials ${ }^{4-5,13-15}$. In the first case, the electronic density is given by $\rho(r, z)=2 r f_{2}(r, z)^{2}$ whereas 
$\rho(r, z)=r\left(f_{1}(r, z)^{2}+2 f_{2}(r, z)^{2}+f_{4}(r, z)^{2}\right)$ in the second case. These states are related to the totally symmetric irreducible representation of the $\mathrm{C}_{\infty \mathrm{V}}$ symmetry group (figure 3 ).

It is possible to study the influence of spin-orbit coupling using the complete $8 \times 8$ Hamiltonian. Figure 4 shows the variations of the energies of the first VB states as a function of $\Delta_{S O}$. The influence of spin-orbit coupling is well approximated by a perturbative calculation. The first order term is equal to zero for the S-shell and yields a splitting proportional to $\Delta_{S O}$ for $F_{z a} \neq 1 / 2$ :

$$
\begin{gathered}
\Delta E_{1}=\int\left(f_{2}(r, z)^{2}-f_{3}(r, z)^{2}\right) \Delta_{2} r d r d z \text { for }\left|\psi_{F_{z a}}^{U}\right\rangle \text { and }\left|\psi_{-F_{z a}}^{L}\right\rangle \\
\Delta E_{1}=-\int\left(f_{2}(r, z)^{2}-f_{3}(r, z)^{2}\right) \Delta_{2} r d r d z \text { for }\left|\psi_{1-F_{z a}}^{U}\right\rangle \text { and }\left|\psi_{F_{z a}-1}^{L}\right\rangle
\end{gathered}
$$

This splitting is almost negligible in the $\mathrm{CB}$ due to the weakness of the $f_{2}(r, z), f_{3}(r, z)$ components. This is exactly the result shown in ref. 5 (table IV). For the VB states, a small energy splitting is expected ${ }^{5}$ : a few meV as indicated by the vertical line in figure 4.

The semi-analytical model presented in this work can be used for any QN in a WZ providing that the geometry and chemical composition of the structure present a ov $_{\text {axis }}$ perpendicular to the (0001) plane. The evaluation of mechanical, piezoelectric, electronic and optical properties is simplified using 2D cylindrical coordinates. An exact analytic expression of the polarization is given with radial and vertical components. The strained $8 \times 8$ k.p Hamiltonian is reduced to $2 \mathrm{D}$ using the total angular momentum representation. When the spin-orbit coupling is neglected, the 2D 4x4 strained Hamiltonians are reduced without any other approximations to $1 \times 1$ and $3 \times 3$ Hamiltonians for the states in the S-shell. The spin-orbit coupling effect can be treated by perturbation theory for the other shells. 


\section{References}

${ }^{1}$ S. L. Chuang and C.S. Chang, Phys. Rev. B54, 2491 (1996).

${ }^{2}$ M. Kumagai, S. L. Chuang and H. Ando, Phys. Rev. B57, 15303 (1998).

${ }^{3}$ F. Mireles and S. E. Ulloa Phys. Rev. B60, 13659 (1999).

${ }^{4}$ A. D. Andreev and E. P. O’Reilly, Phys. Rev. B62, 15851 (2000).

${ }^{5}$ M. Winkelnkemper, A. Schliwa and D. Bimberg, Phys. Rev. B74, 155322 (2006).

${ }^{6}$ L. C. Lew Yan Voon, C. Galeriu, B. Lassen, M. Willatzen and R. Melnik, Appl. Phys. Lett. 87, 041906 (2005).

${ }^{7}$ J. Even, F. Dore, C. Cornet, L. Pedesseau, A. Schliwa and D. Bimberg, Appl. Phys. Lett. 91, $122112(2007)$.

${ }^{8}$ J. Even, F. Dore, C. Cornet, L. Pedesseau, Phys. Rev. B77, 085305 (2008).

${ }^{9}$ I. Vurgaftman and J. R. Meyer, J. Appl. Phys. 94, 7178 (2003).

${ }^{10}$ K. Shimada, T. Sota and K. Suzuki, J. Appl. Phys. 84, 4951 (1998).

${ }^{11}$ K. J. Vahala and P. C. Sercel, Phys. Rev. Lett. 65, 239 (1990).

${ }^{12}$ P. C. Sercel and K. J. Vahala, Phys. Rev. B42, 3690 (1990).

${ }^{13}$ N. Baer, S. Schulz, S. Schumacher, P. Gartner, G. Czycholl and F. Jahnke, Appl. Phys. Lett. 87, 231114 (2005).

${ }^{14}$ S. Schulz, S. Schumacher and G. Czycholl, Phys. Rev B73, 245327 (2006).

${ }^{15}$ V. A. Fonoberov and A. A. Balandin, J. Appl. Phys. 94, 7178 (2003).

${ }^{16}$ N. Baer, S. Schulz, P. Gartner, S. Schumacher, G. Czycholl and F. Jahnke, Phys. Rev B76, 075310 (2007). 


\section{Figure captions}

Figure $1:$ a) Total electric potential for a truncated conic InN/GaN QD (full height, radius and truncation height are respectively equal to 8,4 and 2nm), b) contribution from $\vec{P}_{\text {rad }}$, c) contribution from $\vec{P}_{\text {vert }}$.

Figure 2: Variation of the conduction band (CB) ground state (GS) $\left(F_{z a}=1 / 2\right)$ energy as a function of the truncation height for a truncated conic InN/GaN QD (full height and radius are respectively equal to 8 and $4 \mathrm{~nm}$ ), when the electric potential is: a) calculated with $\vec{P}_{r a d}$ (dash and dotted line), b) calculated with the total polarization $\vec{P}_{W Z}$ (straight line), c) equal to zero (straight line), d) calculated with $\vec{P}_{S P}$ (dotted line) and e) calculated with $\vec{P}_{v e r t}$ (dashed line).

Figure 3 : Isosurfaces of the probability density distributions for the electronic states. The spin-orbit coupling is neglected and the upper $4 \times 4$ block is used but similar results are obtained from the lower block : a) first VB state in the S-shell $\left(F_{z a}=1 / 2\right)$, b) and c) first degenerated VB states in the P-shell $\left.\left(F_{z a}=3 / 2\right), \mathrm{d}\right)$ first CB state in the S-shell $\left(F_{z a}=1 / 2\right)$, e) and f) first degenerated VB states in the P-shell $\left(F_{z a}=3 / 2\right)$.

Figure 4 : Variations of the energies of the first VB states calculated as a function of $\Delta_{S O}$ with the $8 \times 8$ 2D strained Hamiltonian. The $F_{z a}$ parameter (useful when neglecting the spin-orbit coupling) is indicated on the left side while the corresponding total angular moments $\boldsymbol{F}_{z}$ are given on the right side. Splitting are predicted for the P-shell $\left(F_{z a}=3 / 2\right), \mathrm{D}$-shell $\left(F_{z a}=5 / 2\right)$ ... The vertical line corresponds to the $\Delta_{S O}$ value usually used for these nitride compounds. 
FIG. 1.
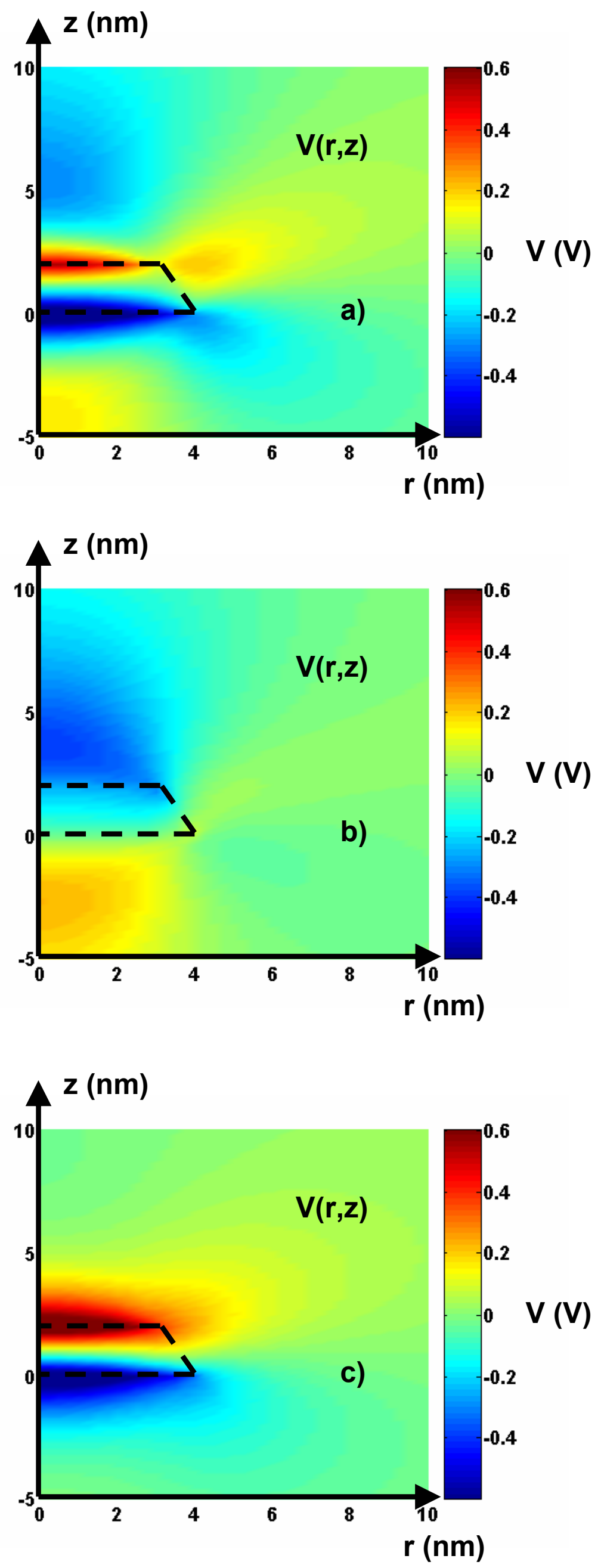
FIG. 2.

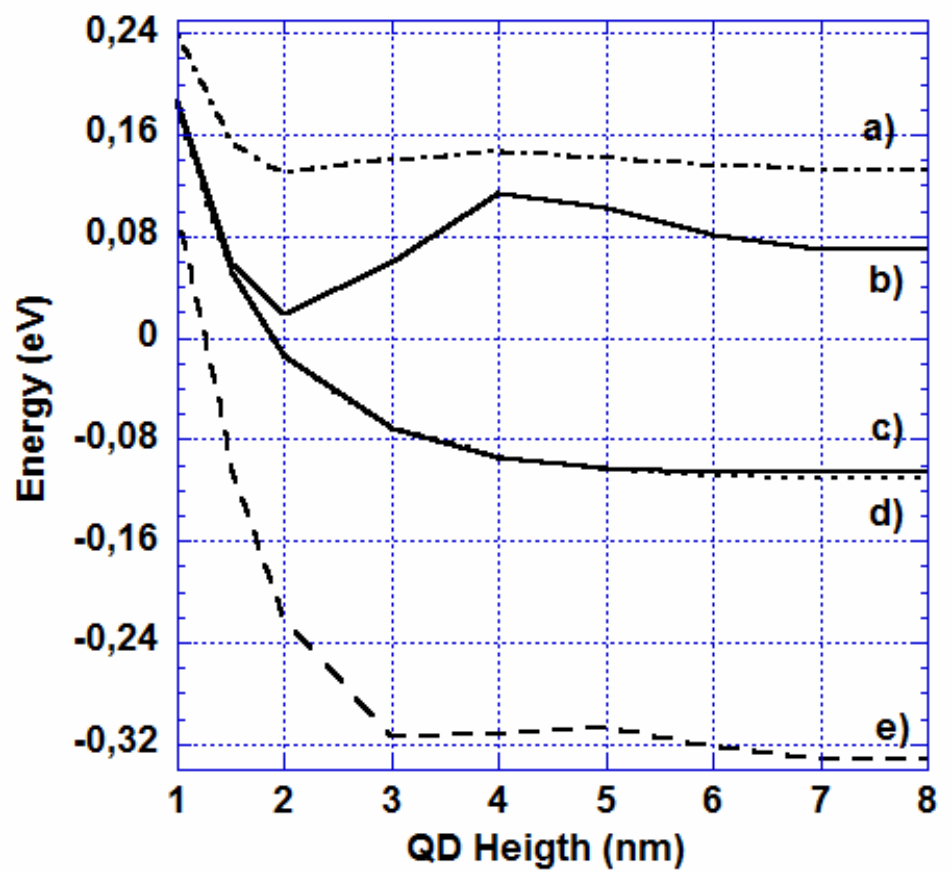

FIG. 3.

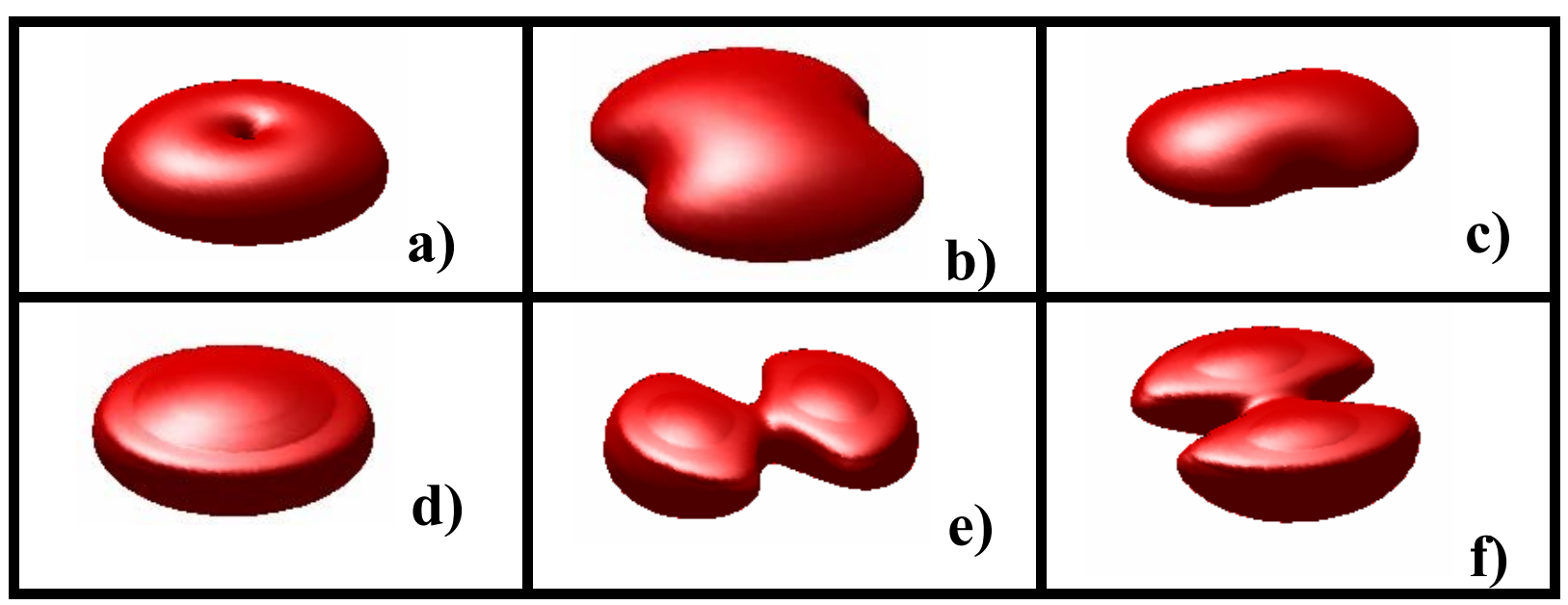


FIG. 4.

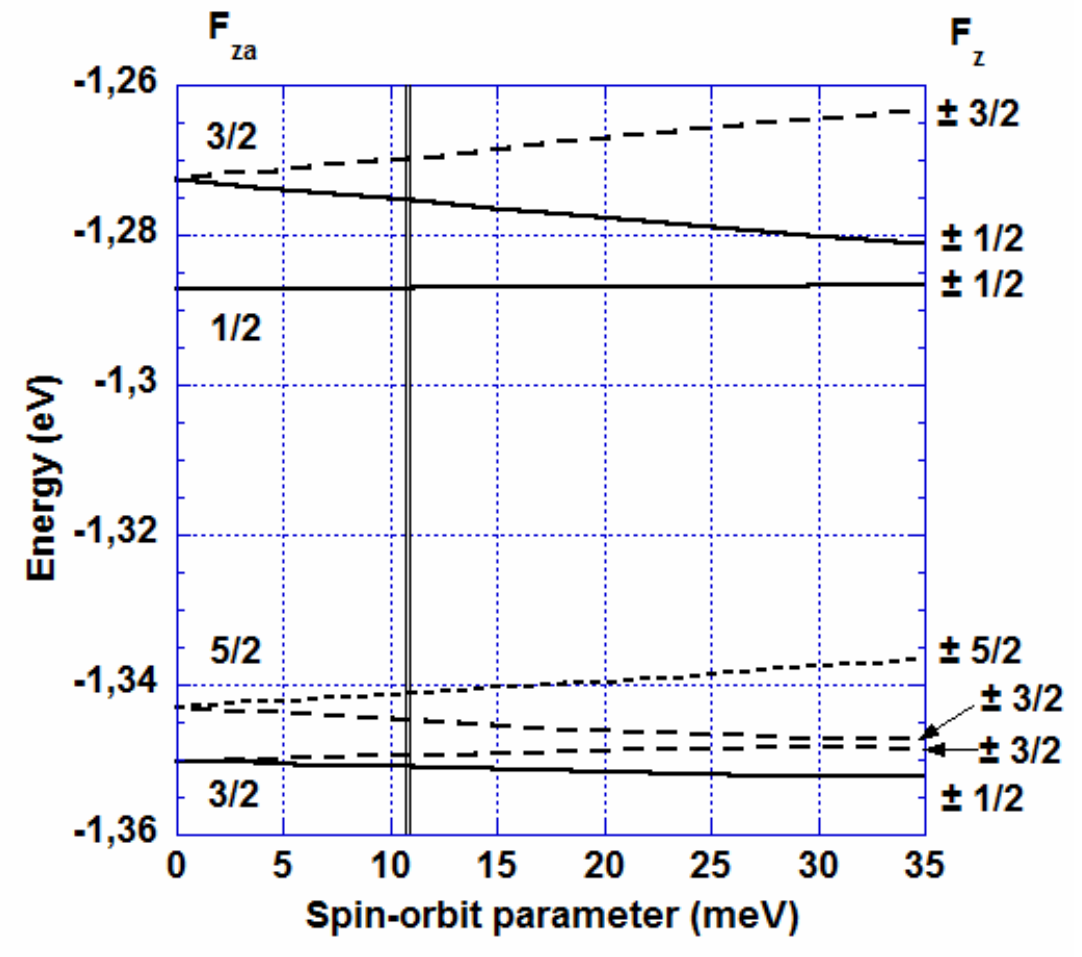

